OPEN ACCESS

Edited by:

Marc Strous,

University of Calgary, Canada

Reviewed by:

A. Murat Eren,

University of Chicago, USA Carolin Regina Löscher, GEOMAR Helmholtz Centre for Ocean Research Kiel, Germany

${ }^{*}$ Correspondence:

Meng Li

limeng848@szu.edu.cn

Specialty section:

This article was submitted to Microbial Physiology and Metabolism, a section of the journal Frontiers in Microbiology

Received: 04 March 2016 Accepted: 06 July 2016

Published: 27 July 2016

Citation:

Li M, Jain S and Dick GJ (2016)

Genomic and Transcriptomic

Resolution of Organic Matter Utilization Among Deep-Sea Bacteria in Guaymas Basin Hydrothermal Plumes. Front. Microbiol. 7:1125. doi: 10.3389/fmicb.2016.01125

\section{Genomic and Transcriptomic Resolution of Organic Matter Utilization Among Deep-Sea Bacteria in Guaymas Basin Hydrothermal Plumes}

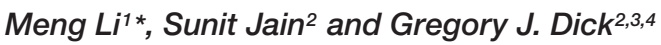 \\ ${ }^{1}$ Institute for Advanced Study, Shenzhen University, Shenzhen, China, ${ }^{2}$ Department of Earth and Environmental Sciences, \\ University of Michigan, Ann Arbor, MI, USA, ${ }^{3}$ Department of Ecology and Evolutionary Biology, University of Michigan \\ Ann Arbor, MI, USA, ${ }^{4}$ Center of Computational Medicine and Bioinformatics, University of Michigan, Ann Arbor, MI, USA
}

Microbial chemosynthesis within deep-sea hydrothermal vent plumes is a regionally important source of organic carbon to the deep ocean. Although chemolithoautotrophs within hydrothermal plumes have attracted much attention, a gap remains in understanding the fate of organic carbon produced via chemosynthesis. In the present study, we conducted shotgun metagenomic and metatranscriptomic sequencing on samples from deep-sea hydrothermal vent plumes and surrounding background seawaters at Guaymas Basin (GB) in the Gulf of California. De novo assembly of metagenomic reads and binning by tetranucleotide signatures using emergent self-organizing maps (ESOM) revealed 66 partial and nearly complete bacterial genomes. These bacterial genomes belong to 10 different phyla: Actinobacteria, Bacteroidetes, Chloroflexi, Deferribacteres, Firmicutes, Gemmatimonadetes, Nitrospirae, Planctomycetes, Proteobacteria, Verrucomicrobia. Although several major transcriptionally active bacterial groups (Methylococcaceae, Methylomicrobium, SUP05, and SAR324) displayed methanotrophic and chemolithoautotrophic metabolisms, most other bacterial groups contain genes encoding extracellular peptidases and carbohydrate metabolizing enzymes with significantly higher transcripts in the plume than in background, indicating they are involved in degrading organic carbon derived from hydrothermal chemosynthesis. Among the most abundant and active heterotrophic bacteria in deep-sea hydrothermal plumes are Planctomycetes, which accounted for seven genomes with distinct functional and transcriptional activities. The Gemmatimonadetes and Verrucomicrobia also had abundant transcripts involved in organic carbon utilization. These results extend our knowledge of heterotrophic metabolism of bacterial communities in deep-sea hydrothermal plumes.

Keywords: metagenomics, metatranscriptomics, heterotrophic metabolism, bacteria, deep-sea hydrothermal plumes 


\section{INTRODUCTION}

Deep-sea hydrothermal vents are typically distributed along the mid-ocean ridges throughout the world's oceans, where hot and chemically reduced hydrothermal vent fluids mix with cold and oxidizing seawater, forming hydrothermal plumes that rise 100 s of meters off the seafloor and disperse 100s of kilometers from their sources. The hydrothermal inputs, such as $\mathrm{H}_{2} \mathrm{~S}$, $\mathrm{H}_{2}, \mathrm{CH}_{4}, \mathrm{NH}_{3}, \mathrm{Mn}^{2+}$ and $\mathrm{Fe}^{2+}$, serve as energy sources that support microbial chemosynthesis (Winn et al., 1986; Deangelis et al., 1993). Evidence has suggested that this chemosynthesis is a significant source of organic carbon to the deep ocean (McCollom, 2000; Lam et al., 2004, 2008; Baker et al., 2012; Lesniewski et al., 2012). Although the global annual biomass production in hydrothermal plumes is a small fraction $\left(\sim 10^{12} \mathrm{~g}\right)$ of total primary production in the oceans, plume chemosynthesis may contribute a substantial fraction of organic carbon in the deep sea (McCollom, 2000). Therefore, understanding the cycling of organic carbon in deep-sea hydrothermal plumes is potentially important in terms of both deep-sea microbiology and microbial food web interactions.

Studies have begun to elucidate the importance and role of microorganisms and their metabolisms that operate within hydrothermal plumes (Lesniewski et al., 2012; Dick et al., 2013). Surveys of small subunit (SSU) ribosomal RNA (rRNA) genes using clone libraries (Sunamura et al., 2004; Dick and Tebo, 2010) and tag pyrosequencing (Sylvan et al., 2012) have revealed the composition of plume microbial communities. Metagenomic and metatranscriptomic results have provided insights into the roles of dominant microorganisms involved in oxidation of sulfur, hydrogen, methane, and ammonia in hydrothermal plumes, such as SUP05 (Anantharaman et al., 2013), Methylococcaceae (Lesniewski et al., 2012; Li et al., 2014a), Marine Group I Thaumarchaea (Baker et al., 2012) and SAR324 (Sheik et al., 2014). Studies also show that rare members of the plume microbial community such as Alteromonadaceae (Li et al., 2014b) and Nitrospiraceae (Baker et al., 2013) are potentially keystone species with roles in iron uptake and nitrite oxidation, respectively. These results have greatly enhanced our knowledge of deep-sea hydrothermal plume microbiology. However, previous studies focused on metabolisms related to autotrophy and inorganic electron donors, and little work has addressed the fate of organic carbon produced via chemosynthesis. Two recent studies presented metagenomic and metatranscriptomic evidence that widespread archaea ( $\mathrm{Li}$ et al., 2015) and Alteromonas bacteria (Baker et al., 2013) play roles in scavenging a variety of organic compounds in the deep sea. Another recent study also inferred a microbial food web in which chemoautotrophy supports and heterotrophy in hydrothermal plumes at the Mid-Cayman Rise (Bennett et al., 2013). However, the broader role of bacteria in processing organic carbon in deepsea hydrothermal plumes, both in terms of specific groups and pathways, remains unclear (Dick et al., 2013).

Guaymas Basin (GB), a submarine depression located on the seabed in the central area of the Gulf of California, hosts an unusual deep-sea hydrothermal system because of its location in a semi-enclosed basin and it proximity to the coast (Lonsdale and Becker, 1985). The ridge axis in GB has been blanketed by a $400 \mathrm{~m}$ layer of organic-rich sediment that chemically modifies hydrothermal fluids as they ascend toward the seafloor (Vondamm et al., 1985). The deep-sea vents inject hydrothermal solutions into the deep waters of a semi-enclosed basin, resulting in a plume where concentrations of methane $(30 \mu \mathrm{M})$, ammonia $(3 \mu \mathrm{M})$, and $\mathrm{Mn}(250 \mathrm{nM})$ are highly enriched over ambient deep sea levels ( $<0.5 \mu \mathrm{M}, 0.25 \mu \mathrm{M}$, and $5 \mathrm{nM}$, respectively) at GB (Dick et al., 2009b). Methane and ammonia are energy sources that fuel substantial and diverse chemoautotrophy that provides a significant source of organic carbon to the deep oceans (Lam et al., 2004; Lesniewski et al., 2012). The objective of this study was to understand organic matter utilization by heterotrophic bacterial communities within GB hydrothermal plumes via shotgun metagenomic and metatranscriptomic sequencing. The results of this study shed light on the ecological and physiological properties of heterotrophic bacteria and highlight their critical role in oceanic carbon cycling.

\section{MATERIALS AND METHODS}

\section{Sample Collection, Extraction of Nucleic Acids, and DNA Sequencing}

Samples from GB were collected on three cruises abroad the R/V New Horizon in 2004 and 2005 as described previously (Dick and Tebo, 2010). Cells for DNA and RNA extraction were filtered directly from the bottle onto a $142 \mathrm{~mm} 0.2 \mathrm{mM}$ polycarbonate filter membrane by $\mathrm{N}_{2}$ gas pressure filtration at $<5$ psi. Filters were preserved immediately in RNA later (Ambion, Austin, TX, USA) as recommended by the manufacturer, incubated at $4^{\circ} \mathrm{C}$ overnight, then stored at $-20^{\circ} \mathrm{C}$ for the duration of the cruise and at $-80^{\circ} \mathrm{C}$ upon return to shore. To minimize the degradation of RNA during collection, samples were kept under in situ conditions (cold and dark) during CTD retrieval and then processed immediately on deck. Although, we cannot exclude the possibility that some changes in the RNA pool occurred during sample collection (it took approximately one hour to recover CTDs from $2000 \mathrm{~m}$ ) and processing, these artifacts should affect all samples evenly and are circumvented to some extent by (i) the vast majority of recovery time is under water at temperatures that do not deviate substantially from those of plume waters, and (ii) the comparative nature of this study (plume versus background). Metadata and chemical/physical characteristics of samples are present in Supplementary Table S1 and more detail as previous reports (Lesniewski et al., 2012; Anantharaman et al., 2013).

DNA was extracted in June of 2012 as described in detail previously (Dick and Tebo, 2010; Lesniewski et al., 2012; Anantharaman et al., 2013; Li et al., 2015). Briefly, $\frac{1}{4}$ filters were cut into small pieces and cells were lysed with bead beating and a chemical solution (300 mM EDTA, $300 \mathrm{mM} \mathrm{NaCl}, 300 \mathrm{mM}$ Tris, pH 7.5, 1.5\% SDS, $50 \mathrm{mM}$ DTT, $0.5 \mathrm{~N}$ acetate and lysozyme). DNA was then purified with Montage PCR purification filter units and eluted in TE buffer. RNA was extracted in June 2012 from 1/4 filters with the mirVana mRNA Isolation kit (Ambion), treated with DNase I to remove DNA, and concentrated and re-purified using the RNeasy MinElute Kit (Qiagen, Valencia, 
CA, USA). Total RNA was quantified by RiboGreen (Invitrogen, Carlsbad, CA, USA). RNA amplification by random priming and complementary DNA (cDNA) synthesis was performed as described previously (Frias-Lopez et al., 2008; Shi et al., 2009; Lesniewski et al., 2012). Shotgun sequencing of DNA and cDNA were performed with Illumina HiSeq2000 PE 100 with HiSeq Cluster Kit v4 (San Diego, CA, USA) at the University of Michigan Sequencing Core.

\section{De Novo Metagenomic Assembly and Gene Annotation}

Metagenomic de novo assembly was performed as described previously (Li et al., 2015). In brief, short DNA reads were dereplicated (100\% identity over 100\% lengths) and trimmed using Sickle ${ }^{1}$. The trimmed and dereplicated pair-end short reads were used for de novo assembly by IDBA-UD with the following parameters: -mink 50, --maxk 92, --step 4 or 6, --min_contig 500. Gene calling and annotations were done using the DOE Joint Genome Institutes (JGI) Integrated Microbial Genomes pipeline $e^{2}$.

\section{Metagenome Binning and Identification of Bins}

Assembled metagenomic sequences were assigned into putative taxonomic groups by binning with emergent self-organizing maps (ESOM) based on the analysis of sequence tetranucleotide frequencies (Dick et al., 2009a; cutoffs: minimum scaffold size $=5 \mathrm{~Kb}$, maximum scaffold size $=10 \mathrm{~Kb}$ ). To identify appropriate taxonomic levels of each genomic bins, we used following order of workflow: (i) identification of SSU rRNA genes on scaffolds by BLASTN (Altschul et al., 1990) against the Silva SSU Database version 115 (Quast et al., 2013); (ii) ESOM binning with reference genomes; (iii) taxonomic clustering of annotated genes by protein UBLAST with the IMG database $(>50 \%)$ of identified open reading frames (Markowitz et al., 2008). Some genomic bins with multiple closely related genomes based on the presence of multiple copies of single-copy conserved genes were further separated by plotting differential coverage of scaffolds and their GC contents (Supplementary Table S2). The completeness and contamination of genomes within bins were estimated by counting single-copy conserved genes using CheckM with default setting, which is an automated method for assessing the quality of microbial genomes recovered from isolates, single cells, and metagenome (Parks et al., 2015; Supplementary Table S3).

\section{Identification of Key Functional Genes}

For genes encoding peptidases and carbohydrate metabolizing enzymes, all annotated genes in bacterial genomes were searched against public databases of peptidases (MEROPS; Rawlings et al., 2014) and Carbohydrate-Active enZYmes (CAZYs; Lombard et al., 2014) with E-value $<10^{-10}$ by BLASTP. To further improve the accuracy after this screening, all hits were also compared to the non-redundant NCBI protein database

${ }^{1}$ https://github.com/najoshi/sickle

${ }^{2}$ http://img.jgi.doe.gov/cgi-bin/w/main.cgi (version 092012). Only those that had top hits to peptidases and carbohydrate metabolizing enzymes [glycoside hydrolases (GHs); polysaccharide lyases (PLs); carbohydrate esterases (CEs); auxiliary activities (AAs)] were considered. The extracellular peptidases were further confirmed based on the identification of extracellular transport signals using SignaIP (Petersen et al., 2011), POSRTb (Yu et al., 2010) or PRED-SIGNAL (Bagos et al., 2009), and these tools are widely used to predict the presence and location of signal peptides and their cleavage sites in amino acid sequences from different organisms. Genes related to carbohydrate metabolizing enzymes were further classified into different groups according the prediction of CAZYs (Lombard et al., 2014).

\section{Analysis of Transcript Abundance of Genomic Bins and Functional Genes}

Abundance of transcripts for each genomic bins or functional genes was determined by mapping all non-rRNA transcripts to the assembled fragments or functional genes using BurrowsWheeler Aligner (BWA) with default settings and normalizing to their sequence length and total number of non-rRNA transcripts in each metatranscriptomic library (Li and Durbin, 2009).

\section{Sequence Alignment and Phylogeny}

Alignment of ribosomal protein S3, 16S rRNA genes were performed by MUSCLE using default parameters followed by manual refinement (Edgar, 2004). Phylogenetic analysis of representative ribosomal protein S3 were inferred by Maximum Likelihood implemented in Mega 6.0 (Tamura et al., 2013) using the Tamura-Nei and passion model after testing by ProTest (Darriba et al., 2011) and bootstrapped 1000 times. The phylogeny of $16 \mathrm{~S}$ rRNA genes were inferred by maximum likelihood implemented in RaxML using the GAMMAGTR algorithm and bootstrap 1000 times in ARB software (Ludwig et al., 2004).

\section{Data Archiving}

Scaffolds from metagenomic assembly are available from DOE JGI-IMG/MEG with Taxon Objects ID 3300001683 and NCBI with accession number LWDU00000000. The original short reads of metagenome and metatranscriptomes are available from NCBI with accession number SRP075597, SRX134768, and SRX134769, respectively.

\section{RESULTS}

\section{Genomic Reconstruction and Identification}

The number of DNA and cDNA short reads from plume and background are listed in Supplementary Table S1. Metagenomic assembly and binning by tetranucleotide frequencies coupled with coverage and GC content resulted in a total of 74 bacterial genomes (Figure 1; Supplementary Table S2). Among them, eight genomes predicted by the CheckM package to have relative high contamination were removed from further analysis. 


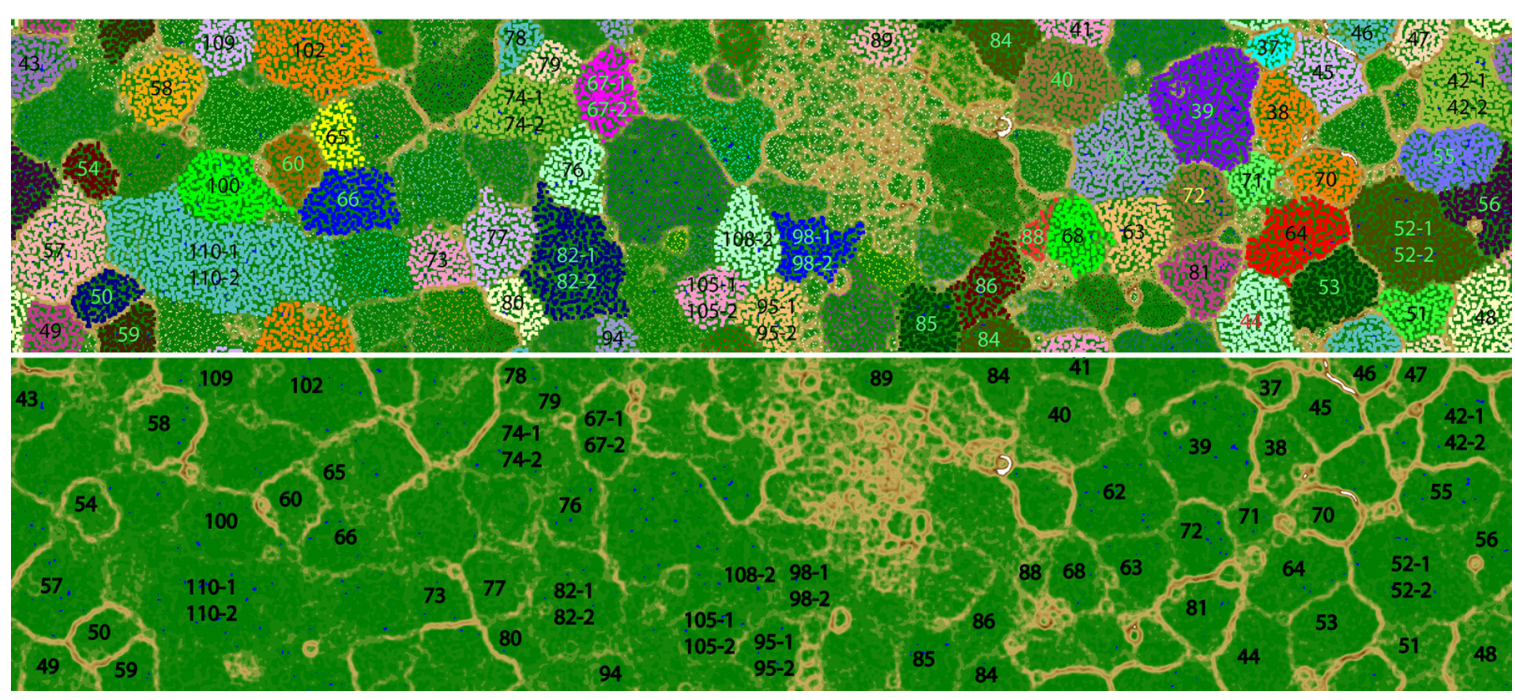

FIGURE 1 | Tetra-nucleotide ESOM binning map of Guaymas Basin plume metagenomic assembly. (Top) Each point on the map represents a contig $(>5 \mathrm{~kb}$ ) or contig fragment generated in silico (5-10 kb). All identified bins are uniquely color coded as indicated. The ESOM map displayed is tiled and torroidal (that is, continuous from top to bottom and left to right). Note that in a few cases (Bin42, Bin52, Bin67, Bin74, Bin82, Bin95, Bin98, Bin105, Bin108, Bin110) multiple closely related bins fall within one large cluster on this map. These bins were delineated based on differential coverage plots. (Bottom) the same ESOM image, without data points shown, highlighting the topographic structure used to delineate bins. Higher "elevations" (brown, white) indicate large tetranucleotide frequency distances between data points, representing boundaries between genomic bins. Lower elevations (green, blue") indicate small tetranucleotide frequency distances between data points, representing the continuity of tetranucleotide frequency space within genomes.

Of the remaining 66 genomes, 53 and 42 of these bacterial genomes were estimated to be $>60$ and $>80 \%$ complete, respectively (Supplementary Table S3). To determine the taxonomic identities of these bacterial genomes, 16S rRNA genes ( $>500 \mathrm{bp}$ ) identified within the genomes (38 genomes) were used for phylogenetic analysis (Figure 2). For remaining genomes (28 genomes), we used phylogeny of ribosomal protein S3 (Supplementary Figure S1) and the binning of reference genomes to confirm their taxonomic identities (Castelle et al., 2013; Hug et al., 2013). These results indicated that the 66 genomes belong to 10 different bacterial phyla, including Actinobacteria (six genomes), Bacteroidetes (seven genomes), Chloroflexi (three genomes), Deferribacteres (three genomes), Firmicutes (one genome), Gemmatimonadetes (one genome), Nitrospirae (two genomes), Planctomycetes (seven genomes), Proteobacteria (Alphaproteobacteria, six genomes; Betaproteobacteria, one genome; Deltaproteobacteria, five genomes; Gammaproteobacteria, 20 genomes), and Verrucomicrobia (four genomes; Supplementary Table S2).

\section{Comparison of Genomic and Transcriptomic Abundance of Bacterial Members}

To compare the genomic and transcriptomic abundance of these genomic bins, DNA and cDNA reads were mapped to the assembled sequences and results were normalized to the sequence length and total number of DNA and cDNA short reads in metagenomic and metatranscriptomic libraries, respectively. From the genomic evidence, the most abundant bacteria in the GB plume included members of SUP05
(Bin82-2, Bin82-1, and Bin94), Actinomycetales (Bin70 and Bin63), Gammaproteobacteria methanotrophs (Bin75, Bin74-2, Bin74-1, Bin66, and Bin65), Planctomycetes (Bin81, Bin102, Bin53, Bin39, Bin44, Bin62, and Bin64), Verrucomicrobia (Bin48, Bin84, Bin49, and Bin40), Acidimicrobium (Bin50), and unknown Gammaproteobacteria (Bin110-2; Figure 3). However, transcriptomic evidence indicated that members of SUP05, methanotrophs, unknown Gammaproteobacteria, Planctomycetes, SAR202 (Bin59), and SAR324 (Bin58) are the most transcriptionally active bacteria groups $\left(>4 \times 10^{6}\right)$, while members of Verrucomicrobia and Acidimicrobium and have relative low transcripts $\left(1.6 \sim 2.7 \times 10^{6}\right)$ in the GB plume and background (Figure 3). Interestingly, most of these bacteria had higher transcript abundances in the plume than in the background with few exceptions from two unknown Gammaproteobacteria (Bin78 and Bin79) and Alteromonadales (Bin95-1 and Bin95-2; Figure 3).

\section{Widespread Occurrence of Extracellular Peptidases in GB Hydrothermal Plume Bacteria}

Of the 66 identified bacterial genomes, 59 genomes from 10 bacterial phyla contained multiple genes encoding extracellular peptidases, ranging from $0.3 / \mathrm{Mb}$ to $9.2 / \mathrm{Mb}$. A member of Gemmatimonadetes (Bin68) had the most abundant extracellular peptidase genes (9.2/Mb), following by members of Erythobacter from Alphaproteobacteria (Bin42-2 and Bin42-1), Rubricoccus and Flavobacteriaceae from Bacteroidetes (Bin46, Bin67-1, and Bin67-2), and Nocardioides from Actinobacteria (Bin88; Figure 4; Supplementary Table S4). Transcripts for extracellular 


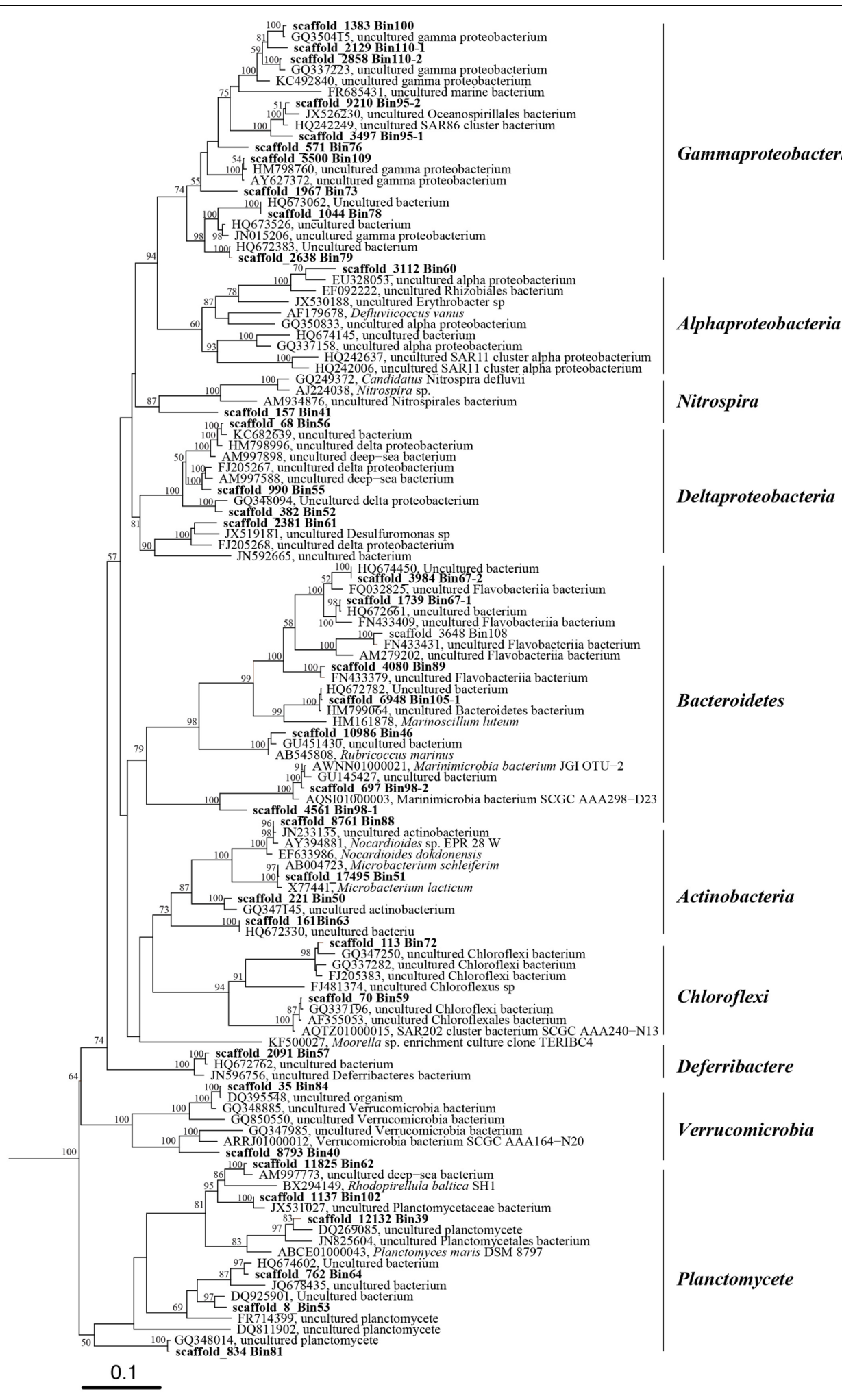

FIGURE 2 | Phylogenetic tree of 16 S rRNA genes from bacterial genomic bins and their closely related sequences in public database. This tree was generated using the maximum likelihood method in the ARB alignment (RAxML, ARB package) and phylogeny software package with 1000 time bootstraps; only bootstraps $>50 \%$ are shown in the tree. 


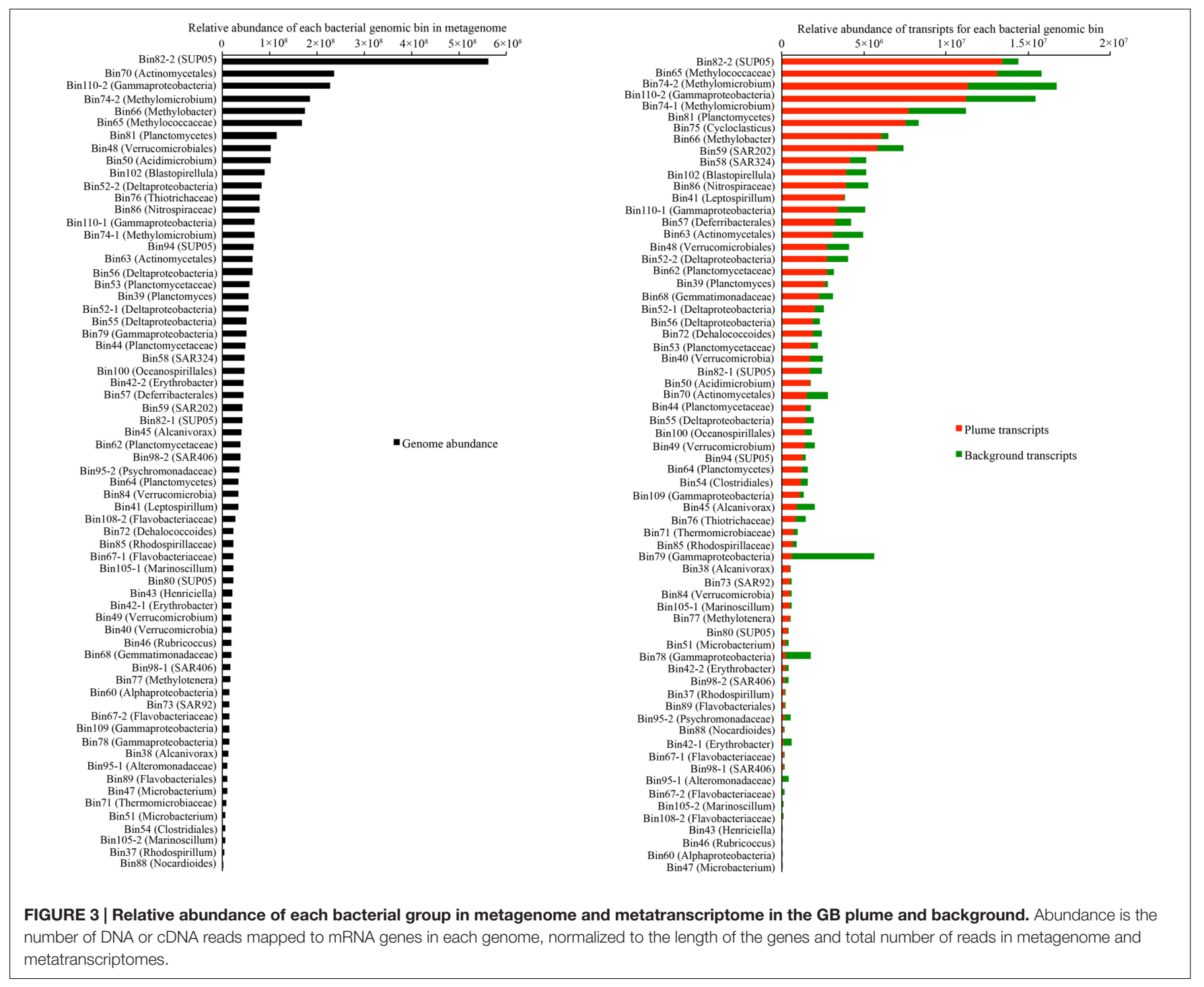

peptidases were also detected from 52 bacterial groups in the GB plume. Among them, members of Methylococcaceae (Bin74-2, Bin66, Bin74-1, and Bin65), Planctomycetes (Bin81, Bin102, Bin44, Bin64, and Bin39), Gemmatimonadetes (Bin68) and unknown Gammproteobacteria (Bin110-2) had the most abundant transcripts for extracellular peptidases in the GB plume, while members of Methylococcaceae (Bin74-2 and Bin74-1) and Gemmatimonadetes (Bin68) also have high extracellular peptidase transcript abundance in the background (Figure 4; Supplementary Table S4).

The detected bacterial extracellular peptidases belong to 58 different families (Figure 5A). Among them, families S01C (DegP serine endopeptidases), S41A (C-terminal processing peptidase), S08A (subtilases), M16B (metalloendopeptidases), and M23B (lysostaphin) were the most abundant in the metatranscriptomes, representing 58.8 and $50.2 \%$ of all bacterial extracellular peptidase transcripts in the GB plume and background, respectively (Figure 5A). These five families of extracellular peptidases are related to serine endopeptidases (S01C, S41A, and S08A) and metalloendopeptidases (M16B and M23B), which cleave peptides at different sites of proteins, suggesting comprehensive protein degradation by deep-sea bacteria and potential use of protein as a growth substrate. Most extracellular peptidases had higher transcript abundance in the plume than in background, with only a few exceptions such as the families M16C (eupitrilysin), S49X (periplasmic serine protease), T02 (isoaspartyl aminopeptidases) and M03A (peptidyl-dipeptidase; Figure 5A).

\section{Ubiquitous Presence of Carbohydrate-Metabolizing Enzymes in GB Hydrothermal Plume Bacteria}

In addition to extracellular peptidases, all bacterial genomes present in here were also searched for carbohydrate-metabolizing enzymes, including GHs, PLs, CEs, and AAs. A total of 65 bacterial genomes contain at least one copy of genes related to carbohydrate-metabolizing enzymes, suggesting 


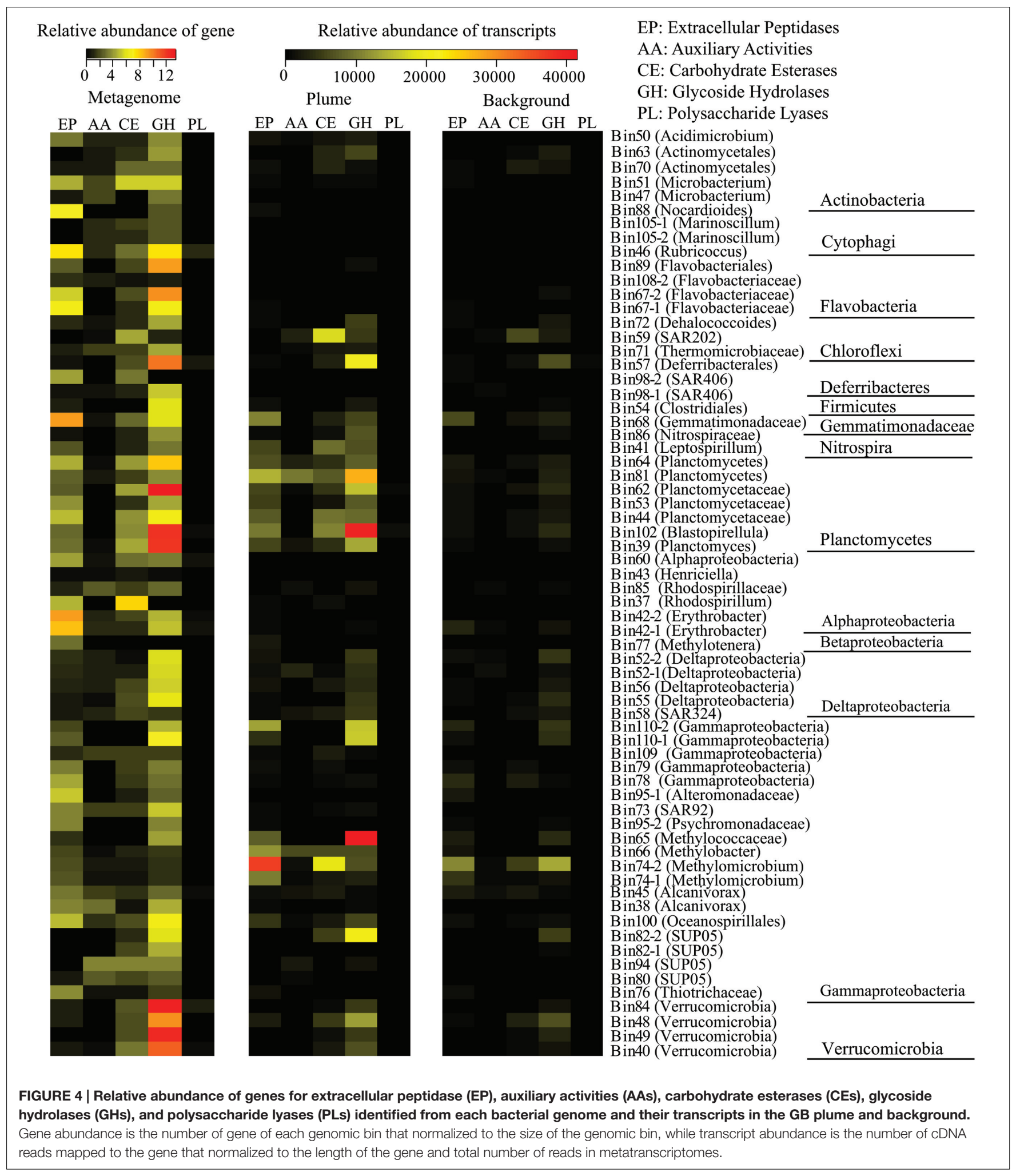

the carbohydrate metabolisms are widespread in deep-sea bacteria identified in the GB hydrothermal plume (Figure 4; Supplementary Table S4). Members from Planctomycetes (Bin62, Bin102, Bin39, Bin64, and Bin44), Verrucomicrobia
(Bin84, Bin49, Bin40, and Bin48), Bacteroidetes (Bin67-2, Bin89, Bin46, Bin108-2, and Bin67-1), Deferribacteres (Bin57) and Gemmatimonadetes (Bin68) have the most abundant genes for $\mathrm{GHs}(>6 / \mathrm{Mb})$, while members of Alphaproteobacteria 


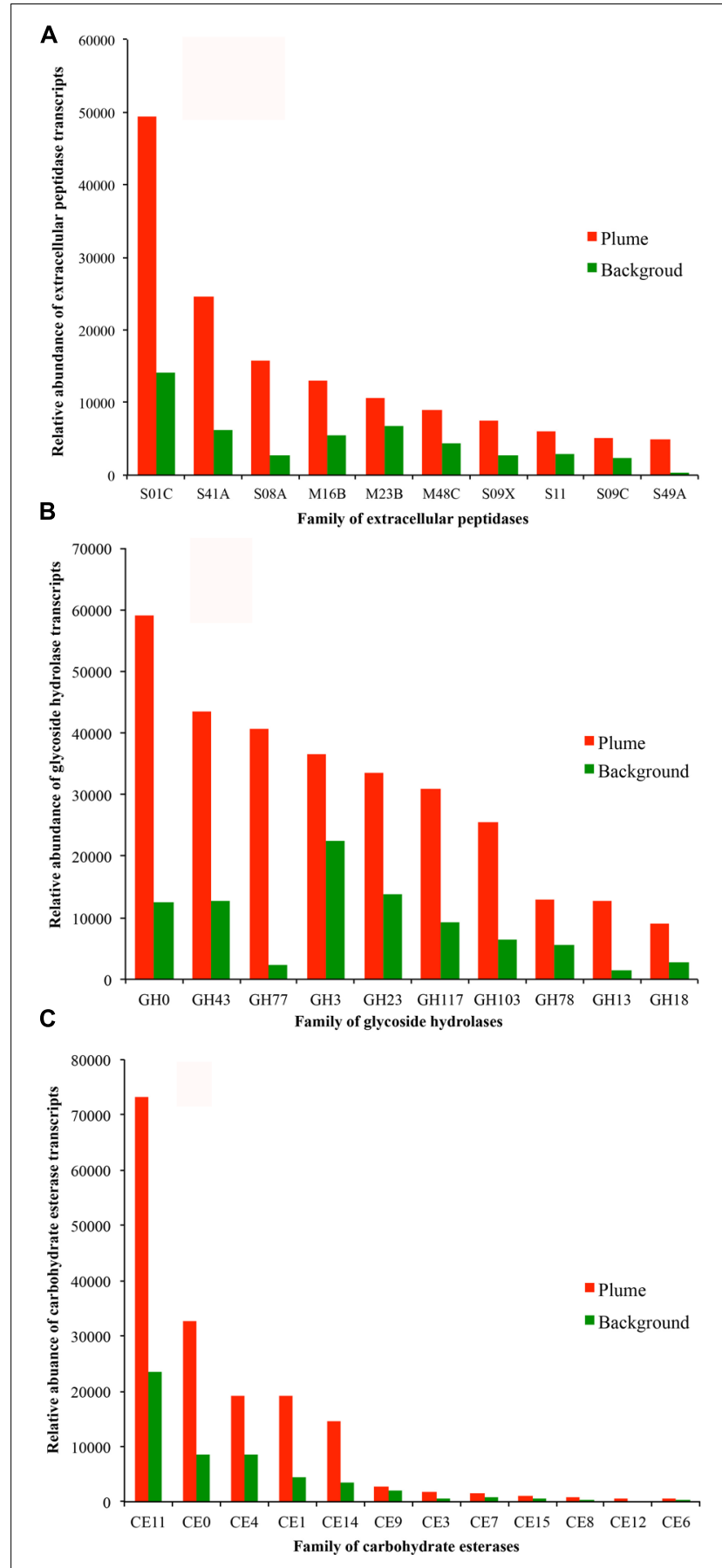

FIGURE 5 | Relative abundance of transcripts at the level of families of extracellular peptidases (A, top 10 families), GHs (B, top 10 families), and CEs (C) identified in the GB plume (red bar) and in the background (green bar). More detailed information is provided in supplementary Figures S2.

(Bin37), Actinobacteria (Bin51), Planctomycetes (Bin39, Bin62, Bin64), and Chloroflexi (Bin59) have high CEs coding gene abundance $(>4 / \mathrm{Mb})$. For PLs and AAs, all bacterial genomes identified here had relatively low gene abundance (Figure 4).
Transcripts for GHs and CEs were detected from 56 and 44 bacteria groups (Figures 5B,C), while only 5 and 31 bacteria groups have transcripts related to PLs and AAs in the GB hydrothermal plume (Supplementary Figure S2). Among these bacteria, members of Methylococcaceae (Bin65), Planctomycetes (Bin102, Bin81), SUP05 (Bin82-2), Deferribacteres (Bin57) and Verrucomicrobia (Bin48) have the most abundant transcripts for GHs, while members of Methylococcaceae (Bin74-2), SAR202 (Bin59), and Planctomycetes (Bin102 and Bin44) have high CE transcripts. Furthermore, GHs and CEs have much higher transcript abundance than those related to PLs and AAs (Figure 4; Supplementary Table S4).

Further analysis demonstrates that families of GH0, GH43, GH77, GH3, GH23, GH117, and GH103 for GH are the most abundant in the metatranscriptomes, representing 71.8 and $70.2 \%$ of all bacterial GH transcripts in the GB plume and background (Figure 5B). These dominant families are related to aldose 1-epimerases, arylsulfatases, sulfatases, beta-lactamases, beta- $N$-acetylglucosaminidases, lytic transglycosylases, $N$-acetylbeta-hexosaminidases, and many uncharacterized GHs. While for CEs, families of CE11 and CE0 are the most abundant in the metatranscriptomes, responding to 63.0 and $60.5 \%$ of all bacterial CE transcripts in the GB plume and in the background (Figure 5C). Finally, similar with the extracellular peptidases, all detected families of GHs and CEs have much higher transcripts in the plume than in the background (Figure 5).

\section{Organic Matter Utilization of Planctomycetes, Gemmatimonadetes, and Verrucomicrobia in Deep-Sea Hydrothermal Plumes}

To better understand the most active heterotrophic bacteria, the extracellular peptidases and carbohydrate-metabolizing enzymes in Planctomycetes, Gemmatimonadetes, and Verrucomicrobia were characterized further. Members of Planctomycetes are the most abundant and transcriptionally active heterotrophic bacteria in the GB hydrothermal plume (Supplementary Figure S3). Seven near-complete genomes of Planctomycetes with distinct genomic and transcriptomic abundance were identified (Figure 6). These seven genomes contained a total of 122 extracellular peptidase genes from 33 different families. Among them, transcripts for 27 families were detected, representing $27.9 \%$ of all bacterial extracellular peptidase transcripts in the GB plume (Figure 6A). For carbohydrate metabolizing enzymes, a total of 369 genes for 29 different families of GHs and 134 of $\mathrm{CE}$ genes belonging to 10 families were also identified in the seven Planctomycete genomes, representing 31.9 and $26.6 \%$ of all bacterial transcripts for GHs and CEs in the GB plume, respectively (Figure 6B). Similarly, transcripts involved in PLs and AAs identified in members of Planctomycetes also accounted for 35.4 and $69.3 \%$ of all related bacterial transcripts in the GB plume. These results indicate that members of Planctomycetes are involved in scavenging various organic matter in the GB plume. Interestingly, these Planctomycetes bacteria also have many genes and transcripts involved in the hydrolysis of sulfate esters, such as arylsulfatase, choline sulfatases and sulfatases, indicating 


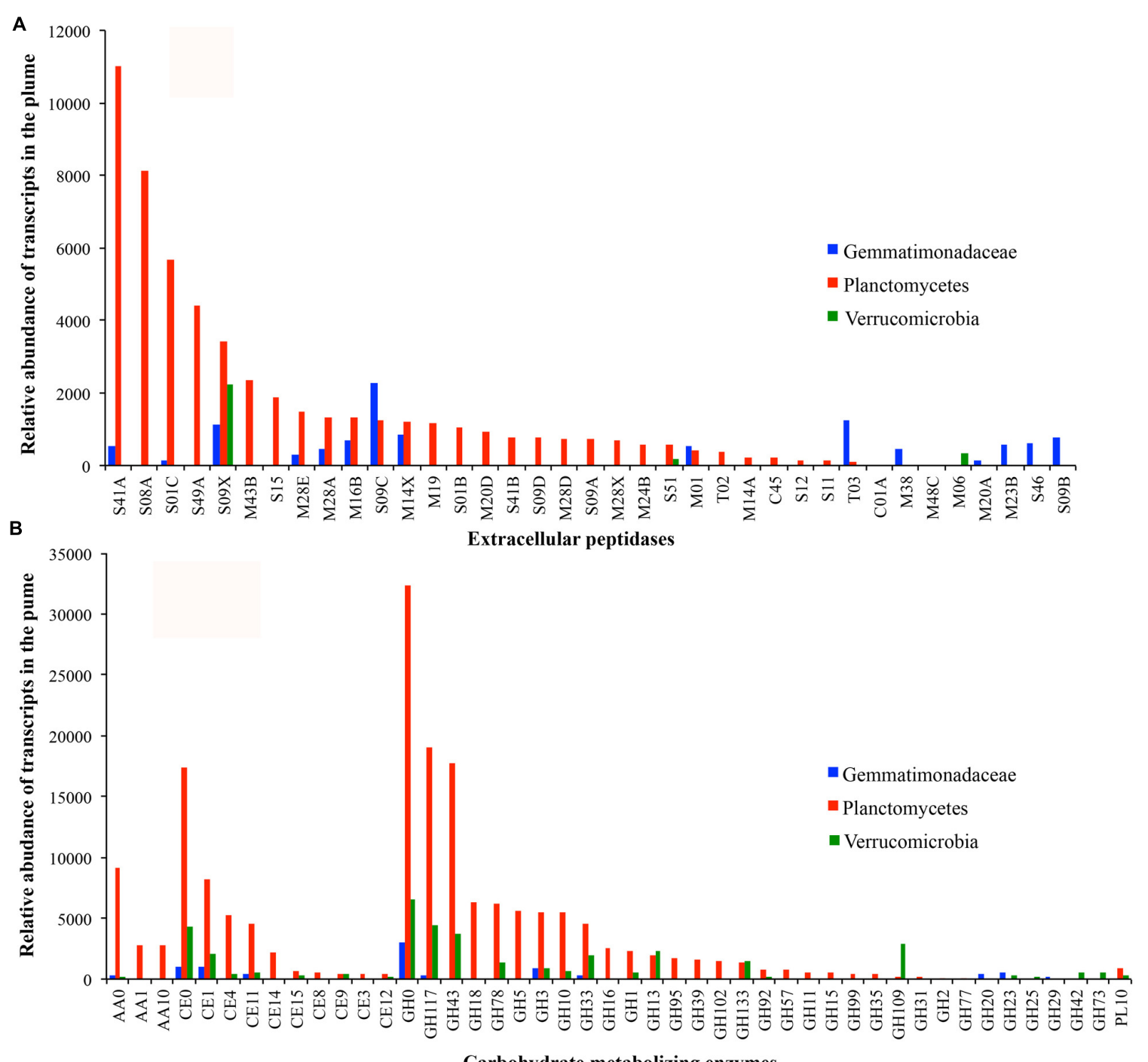

Carbohydrate metabolizing enzymes

FIGURE 6 | Transcript abundance of extracellular peptidases (A) and carbohydrate metabolizing enzymes (B) in bacteria of Gemmatimonadetes (blue), Plantomycetes (red), and Verrucomicrobia (green) in the GB plume.

Planctomycetes also respond for the utilization of organic sulfur compounds in the deep-sea (Supplementary Figure S4).

Only one genome related to Gemmatimonadetes was identified in the GB hydrothermal plume. This Gemmatimonadetes genome was estimated to be $85.8 \%$ complete and contained high abundance of genes for extracellular peptidases (the most of all bacterial genomes recovered) and carbohydrate metabolizing enzymes (Figure 4). Furthermore, this deep-sea Gemmatimonadetes bacterium also had high transcript abundance for carbohydrate metabolizing enzymes and extracellular peptidases, accounting for 1.5 and 5.5\% of all bacterial carbohydrate metabolizing enzyme and extracellular peptidase transcripts, respectively (Figure 6A). For members of Verrucomicrobia, four genomes were identified in the GB hydrothermal plume, ranging in completeness from 44.4 to $99.8 \%$. In these Verrucomicrobia, only eight extracellular peptidase genes were identified, but 59 genes encoding carbohydrate metabolizing enzymes were found, the most of all bacteria identified in this study. In addition, Verrucomicrobia bacteria had high abundance of transcripts for carbohydrate metabolizing enzymes, but relative lower transcripts for extracellular peptidases (Figures 4 and 6).

\section{DISCUSSION}

In the GB hydrothermal plume, the dominant active methanotrophs and chemolithotrophs produce labile organic matter that may support heterotrophic microorganisms (Baker et al., 2012; Lesniewski et al., 2012; Anantharaman et al., 2013; Li et al., 2014b). A recent study has reported the heterotrophic metabolisms of deep-sea archaea, indicating the critical roles of 
ubiquitous archaea in scavenging various organic matter (Li et al., 2015). To understand the bacterial heterotrophic metabolisms, we have reconstructed a total of 66 bacterial genomes from the GB hydrothermal plume through the combined use of shotgun metagenomic sequencing, de novo genomic assembly, tetranucleotide signature ESOM binning, and manual curation (Figure 1; Supplementary Table S2). The identified 66 bacterial genomes affiliate to 10 different bacterial phyla, indicating a diverse community structure and greatly expanding the genomic coverage of GB plume microorganisms. Furthermore, most of reconstructed bacterial genomes are nearly complete, including many uncharacterized deep-sea bacteria, such as members of Deferribacteres (Bin98-1, Bin98-2, and Bin57) and unknown Deltaproteobacteria (Bin52-1, Bin52-2, Bin55, Bin56, and Bin61; Supplementary Table S3). Thus, the data presented here provide substantial genomic and transcriptomic information to understand the function of bacteria in deep-sea hydrothermal plumes. We note that the metatranscriptomic data presented in this paper are in terms of relative abundance, thus we are unable to make inferences regarding levels of gene expression. Rather our results are in terms of relative abundance of transcripts, which provides a valuable measure of the relative contributions of genes and organisms to the pool of community transcripts.

Although the microbial community structures in the GB hydrothermal plume are similar to these in surrounding background waters (Dick and Tebo, 2010; Lesniewski et al., 2012), most of the bacteria in the plume had higher relative abundance of transcripts than in background, suggesting bacteria in the GB plume are stimulated by abundant substrates (Figure 3). Consistent with previous results, both genomic and transcriptomic evidence show that the most abundant and transcriptionally active bacterial groups were members of SUP05, methanotrophs, and SAR324, which display evidence of chemolithoautotrophic metabolisms (Baker et al., 2012; Lesniewski et al., 2012; Anantharaman et al., 2013; Li et al., 2014a; Sheik et al., 2014). However, the presence of genes encoding extracellular peptidases and carbohydrate metabolizing enzymes in most of bacterial genomes in the GB plume suggests that the potential for organic matter utilization in the deep-sea bacteria is also widespread (Figure 4). Taken together with the results of archaea in GB (Li et al., 2015), the utilization of organic matter is one of most common microbial metabolisms in the GB hydrothermal plume. Furthermore, transcriptomic evidence indicated that 66 bacterial groups from 10 bacterial phyla contain transcripts for different families of extracellular peptidases and carbohydrate metabolizing enzymes, suggesting these bacteria from different phyla are transcriptionally active for different organic matter utilization in the GB hydrothermal plume. Given the predominant chemolithoautotrophic metabolisms in the GB hydrothermal plume, the much higher abundance of transcripts of extracellular peptidases and carbohydrate metabolizing enzymes in the plume than in the background support that these bacteria may be degrading organic carbon derived from hydrothermal chemosynthesis.

The Planctomycetes are a bacterial phylum that is ubiquitous in natural environments. All currently described pure cultures of Planctomycetes are aerobic heterotrophs (the members of
Brocadiaceae that conduct anaerobic ammonia oxidation are still not purified) capable of growth on several sugars and sugar alcohols, such as glucose, fructose, mannitol, xylose, ribose, and fucose (Youssef and Elshahed, 2014). Many grow exceptionally well on specific substrates, such as $\mathrm{N}$-acetylglucosamine (NAG) presented as a side chain on mucin and chondroitin sulfate in nature. Furthermore, several described Planctomycetes species can metabolize polysaccharides, including starch, gelatin, and carboxymethyl cellulose (Schlesner et al., 2004; Kulichevskaya et al., 2009; Bondoso et al., 2011; Kulichevskaya et al., 2012). This preference for monomers and sulfated polymers has lead to suggestions that degradation of sulfated polymeric carbon, e.g., marine snow, is a natural role for Planctomycetes in various habitats (Glöckner et al., 2003; Woebken et al., 2007). Consistent with previous results, seven genomes of Planctomycetes identified in the GB hydrothermal plume contain abundant genes and transcripts for extracellular peptidases and carbohydrate metabolizing enzymes, particularly genes related to sulfatases, suggesting they perform important roles in organic carbon cycling in the GB plume (Figure 6).

Bacteria belonging to phylum Gemmatimonadetes have been identified as one of the top nine phyla found in soils, comprising $\sim 2 \%$ of soil bacterial communities (Bossio et al., 1998; DeBruyn et al., 2011). Despite their frequency and persistent abundance in soils, information about this phylum in marine environments is quite limited. Currently, only few representatives from this phylum have been isolated and partially characterized (Joseph et al., 2003; Zhang et al., 2003; Davis et al., 2005). Members of Gemmatimonadetes are aerobic heterotrophs capable of utilization of multiple substrates, including east extract, polypepton, succinate, acetate, gelatin, and benzoate (Zhang et al., 2003). A recent study reported five genomes of Gemmatimonadetes from estuary sediments and found that members of Gemmatimonadetes contain a large number of genes of carbohydrate hydrolysis and protein degradation (Baker et al., 2015). Here, we have reconstructed a nearly complete (85.8\%) genome of Gemmatimonadetes from deep oceans, which has the most abundant extracellular peptidase genes $(9.2 / \mathrm{Mb})$. Together with results from transcriptomes, as well as carbohydrate metabolizing enzymes, the data presented here strongly support Gemmatimonadetes bacteria as key mediators of the organic carbon cycle in the GB hydrothermal plumes (Figure 6).

The third interesting bacteria group are members belonging to the phylum of Verrucomicrobia, which are widely detected in different freshwater and marine habits (Wagner and Horn, 2006). Previous studies have confirmed that members of Verrucomicrobia are capable of heterotrophic, carbohydrate-degrading metabolisms (Arnosti, 2011; Steen et al., 2012; Cardman et al., 2014). The genomic evidence from a metagenome in Baltic seawater indicated that the genome of Verrucomicrobia bacteria encodes a diversity of GHs that likely allow degradation of various complex carbohydrates, such as cellulose, mannan, xylan, chitin, and starch (Herlemann et al., 2013). A recent report also proposed Verrucomicrobia are involved in polysaccharide hydrolysis in the water column and sediment 
(Cardman et al., 2014). Here, the four genomes of Verrucomicrobia contain diverse GH and CE genes, which also have relative high transcripts in the GB hydrothermal plume (Figure 6B), implying important roles of these microorganisms in the deep-sea carbon cycle.

\section{CONCLUSION}

In summary, a total of 66 bacterial genomes from 10 different phyla in the GB hydrothermal plume were reconstructed through a combination with high-throughput sequencing, de novo genomic assembly and tetranucleotide binning. The widespread presence of extracellular peptidase and carbohydrate metabolizing enzyme genes in most of bacterial genomes suggests that the utilization of organic carbon is a common microbial metabolism in the GB hydrothermal plume. Furthermore, the higher relative abundance of transcripts of extracellular peptidases and carbohydrate metabolizing enzymes in the plume than in background indicate these bacteria might be involved in degradation of organic carbon derived from hydrothermal chemosynthesis. Hence, primary and secondary production in hydrothermal plumes may be tightly coupled. Although the mechanism of trophic transfer is unknown, the presence of abundant viruses that infect the autotrophs in GB and other hydrothermal plumes (Anantharaman et al., 2014) suggests that viral lysis is one potential source of carbon for heterotrophs. Among the most abundant and active heterotrophic bacteria in deep-sea hydrothermal plumes are members of the Planctomycetes, Gemmatimonadetes, and Verrucomicrobia. These results extend our knowledge of

\section{REFERENCES}

Altschul, S. F., Gish, W., Miller, W., Myers, E. W., and Lipman, D. J. (1990). Basic local alignment search tool. J. Mol. Biol. 215, 403-410. doi: 10.1016/S00222836(05)80360-2

Anantharaman, K., Breier, J. A., Sheik, C. S., and Dick, G. J. (2013). Evidence for hydrogen oxidation and metabolic plasticity in widespread deep-sea sulfur-oxidizing bacteria. Proc. Natl. Acad. Sci. U.S.A. 110, 330-335. doi: 10.1073/pnas. 1215340110

Anantharaman, K., Duhaime, M. B., Breier, J. A., Wendt, K. A., Toner, B. M., and Dick, G. J. (2014). Sulfur oxidation genes in diverse deep-sea viruses. Science 344, 757-760. doi: 10.1126/science. 1252229

Arnosti, C. (2011). Microbial extracellular enzymes and the marine carbon cycle. Annu. Rev. Mar. Sci. 3, 401-425. doi: 10.1146/annurev-marine-120709-142731

Bagos, P. G., Tsirigos, K. D., Plessas, S. K., Liakopoulos, T. D., and Hamodrakas, S. J. (2009). Prediction of signal peptides in archaea. Protein Eng. Des. Sel. 22, 27-35. doi: 10.1093/protein/gzn064

Baker, B. J., Lazar, C. S., Teske, A. P., and Dick, G. J. (2015). Genomic resolution of linkages in carbon, nitrogen, and sulfur cycling among widespread estuary sediment bacteria. Microbiome 3, 14. doi: 10.1186/s40168-015-0077-6

Baker, B. J., Lesniewski, R. A., and Dick, G. J. (2012). Genome-enabled transcriptomics reveals archaeal populations that drive nitrification in a deepsea hydrothermal plume. ISME J. 6, 2269-2279. doi: 10.1038/ismej.2012.64

Baker, B. J., Sheik, C. S., Taylor, C. A., Jain, S., Bhasi, A., Cavalcoli, J. D., et al. (2013). Community transcriptomic assembly reveals microbes that contribute to deep-sea carbon and nitrogen cycling. ISME J. 7, 1962-1973. doi: 10.1038/ismej.2013.85

Bennett, S. A., Coleman, M., Huber, J. A., Reddington, E., Kinsey, J. C., McIntyre, C., et al. (2013). Trophic regions of a hydrothermal plume dispersing heterotrophic metabolism of bacterial communities within deep-sea hydrothermal plumes, highlighting their critical role in organic carbon cycling in the deep-sea hydrothermal plumes.

\section{AUTHOR CONTRIBUTIONS}

ML and GD designed the study; ML and SJ performed the genomic analyses. All authors wrote the manuscript and the Supplementary Materials.

\section{ACKNOWLEDGMENTS}

This project was funded in part by the Gordon and Betty Moore Foundation Grant GBMF2609, National Science Foundation Grants OCE1038006 (GD) and National Natural Science Foundation of China (grant no. 41506163), Natural Science Foundation of Guangdong Province (grant no. 2014A030310056), and Shenzhen City (grant no. JCY20140828163633985 and KQCX2015032416053646; ML). DNA sequencing was conducted at the University of Michigan DNA Sequencing Core.

\section{SUPPLEMENTARY MATERIAL}

The Supplementary Material for this article can be found online at: http://journal.frontiersin.org/article/10.3389/fmicb. 2016.01125

away from an ultramafic-hosted vent-system: Von Damm vent-site, MidCayman Rise. Geochem. Geophys. Geosyst. 14, 317-327. doi: 10.1002/ggge.20063 Bondoso, J., Albuquerque, L., Nobre, M. F., Lobo-da-Cunha, A., da Costa, M. S., and Lage, O. M. (2011). Aquisphaera giovannonii gen. nov., sp nov., a planctomycete isolated from a freshwater aquarium. Int. J. Syst. Evol. Microbiol. 61, 2844-2850. doi: 10.1099/ijs.0.027474-0

Bossio, D. A., Scow, K. M., Gunapala, N., and Graham, K. J. (1998). Determinants of soil microbial communities: effects of agricultural management, season, and soil type on phospholipid fatty acid profiles. Microb. Ecol. 36, 1-12. doi: $10.1007 / \mathrm{s} 002489900087$

Cardman, Z., Arnosti, C., Durbin, A., Ziervogel, K., Cox, C., Steen, A. D., et al. (2014). Verrucomicrobia are candidates for polysaccharide-degrading bacterioplankton in an arctic fjord of Svalbard. Appl. Environ. Microbiol. 80, 3749-3756. doi: 10.1128/AEM.00899-14

Castelle, C. J., Hug, L. A., Wrighton, K. C., Thomas, B. C., Williams, K. H., Wu, D. Y., et al. (2013). Extraordinary phylogenetic diversity and metabolic versatility in aquifer sediment. Nat. Commun. 4, 2120. doi: $10.1038 /$ ncomms3120

Darriba, D., Taboada, G. L., Doallo, R., and Posada, D. (2011). ProtTest 3: fast selection of best-fit models of protein evolution. Bioinformatics 27, 1164-1165. doi: 10.1093/bioinformatics/btr088

Davis, K. E. R., Joseph, S. J., and Janssen, P. H. (2005). Effects of growth medium, inoculum size, and incubation time on culturability and isolation of soil bacteria. Appl. Environ. Microbiol. 71, 826-834. doi: 10.1128/AEM.71.2.826834.2005

Deangelis, M. A., Lilley, M. D., Olson, E. J., and Baross, J. A. (1993). Methane oxidation in deep-sea hydrothermal plumes of the endeavor segment of the Juan-de-Fuca Ridge. Deep Sea Res. Part 1 Oceanogr. Res. Pap. 40, 1169-1186. doi: $10.1121 / 1.3624816$ 
DeBruyn, J. M., Nixon, L. T., Fawaz, M. N., Johnson, A. M., and Radosevich, M. (2011). Global biogeography and quantitative seasonal dynamics of Gemmatimonadetes in soil. Appl. Environ. Microbiol. 77, 6295-6300. doi: 10.1128/AEM.05005-11

Dick, G. J., Anantharaman, K., Baker, B. J., Li, M., Reed, D. C., and Sheik, C. S. (2013). The microbiology of deep-sea hydrothermal vent plumes: ecological and biogeographic linkages to seafloor and water column habitats. Front. Microbiol. 4:124. doi: $10.3389 /$ fmicb.2013.00124

Dick, G. J., Andersson, A. F., Baker, B. J., Simmons, S. L., Thomas, B. C., Yelton, A. P., et al. (2009a). Community-wide analysis of microbial genome sequence signatures. Genome Biol. 10:R85. doi: 10.1186/gb-2009-10-8-r85

Dick, G. J., Clement, B. G., Webb, S. M., Fodrie, F. J., Bargar, J. R., and Tebo, B. M. (2009b). Enzymatic microbial Mn(II) oxidation and Mn biooxide production in the Guaymas Basin deep-sea hydrothermal plume. Geochim. Cosmochim. Acta 73, 6517-6530. doi: 10.1016/j.gca.2009.07.039

Dick, G. J., and Tebo, B. M. (2010). Microbial diversity and biogeochemistry of the Guaymas Basin deep-sea hydrothermal plume. Environ. Microbiol. 12, 1334-1347. doi: 10.1111/j.1462-2920.2010.02177.x

Edgar, R. C. (2004). MUSCLE: multiple sequence alignment with high accuracy and high throughput. Nucleic Acids Res. 32, 1792-1797. doi: 10.1093/nar/gkh340

Frias-Lopez, J., Shi, Y., Tyson, G. W., Coleman, M. L., Schuster, S. C., Chisholm, S. W., et al. (2008). Microbial community gene expression in ocean surface waters. Proc. Natl. Acad. Sci. U.S.A. 105, 3805-3810. doi: 10.1073/pnas.0708897105

Glöckner, F. O., Kube, M., Bauer, M., Teeling, H., Lombardot, T., Ludwig, W., et al. (2003). Complete genome sequence of the marine planctomycete Pirellula sp strain 1. Proc. Natl. Acad. Sci. U.S.A. 100, 8298-8303. doi: 10.1073/pnas.1431443100

Herlemann, D. P. R., Lundin, D., Labrenz, M., Jurgens, K., Zheng, Z. L., Aspeborg, H., et al. (2013). Metagenomic de novo assembly of an aquatic representative of the verrucomicrobial class Spartobacteria. MBio 4:e00569-12. doi: $10.1128 / \mathrm{mBio} .00569-12$

Hug, L. A., Castelle, C. J., Wrighton, K. C., Thomas, B. C., Sharon, I., Frischkorn, K. R., et al. (2013). Community genomic analyses constrain the distribution of metabolic traits across the Chloroflexi phylum and indicate roles in sediment carbon cycling. Microbiome 1, 22. doi: 10.1186/2049-2618-1-22

Joseph, S. J., Hugenholtz, P., Sangwan, P., Osborne, C. A., and Janssen, P. H. (2003). Laboratory cultivation of widespread and previously uncultured soil bacteria. Appl. Environ. Microbiol. 69, 7210-7215. doi: 10.1128/AEM.69.12.72107215.2003

Kulichevskaya, I. S., Baulina, O. I., Bodelier, P. L. E., Rijpstra, W. I. C., Damste, J. S. S., and Dedysh, S. N. (2009). Zavarzinella formosa gen. nov., sp nov., a novel stalked, Gemmata-like planctomycete from a Siberian peat bog. Int. J. Syst. Evol. Microbiol. 59, 357-364. doi: 10.1099/ijs.0.002378-0

Kulichevskaya, I. S., Serkebaeva, Y. M., Kim, Y., Rijpstra, W. I. C., Damste, J. S. S., Liesack, W., et al. (2012). Telmatocola sphagniphila gen. nov., sp nov., a novel dendriform planctomycete from northern wetlands. Front. Microbiol. 3:146. doi: $10.3389 /$ fmicb. 2012.00146

Lam, P., Cowen, J. P., and Jones, R. D. (2004). Autotrophic ammonia oxidation in a deep-sea hydrothermal plume. FEMS Microbiol. Ecol. 47, 191-206. doi: 10.1016/S0168-6496(03)00256-3

Lam, P., Cowen, J. P., Popp, B. N., and Jones, R. D. (2008). Microbial ammonia oxidation and enhanced nitrogen cycling in the Endeavour hydrothermal plume. Geochim. Cosmochim. Acta 72, 2268-2286. doi: 10.1016/S01686496(03)00256-3

Lesniewski, R. A., Jain, S., Anantharaman, K., Schloss, P. D., and Dick, G. J. (2012). The metatranscriptome of a deep-sea hydrothermal plume is dominated by water column methanotrophs and lithotrophs. ISME J. 6, 2257-2268. doi: 10.1038/ismej.2012.63

Li, H., and Durbin, R. (2009). Fast and accurate short read alignment with Burrows-Wheeler transform. Bioinformatics 25, 1754-1760. doi: 10.1093/bioinformatics/btp324

Li, M., Baker, B. J., Anantharaman, K., Jain, S., Breier, J. A., and Dick, G. J. (2015). Genomic and transcriptomic evidence for scavenging of diverse organic compounds by widespread deep-sea archaea. Nat. Commun. 6, 8933. doi: 10.1038/ncomms 9933

Li, M., Jain, S., Baker, B. J., Taylor, C., and Dick, G. J. (2014a). Novel hydrocarbon monooxygenase genes in the metatranscriptome of a natural deep-sea hydrocarbon plume. Environ. Microbiol. 16, 60-71. doi: 10.1111/14622920.12182

Li, M., Toner, B. M., Baker, B. J., Breier, J. A., Sheik, C. S., and Dick, G. J. (2014b). Microbial iron uptake as a mechanism for dispersing iron from deep-sea hydrothermal vents. Nat. Commun. 5, 3192. doi: 10.1038/ncomms4192

Lombard, V., Golaconda Ramulu, H., Drula, E., Coutinho, P. M., and Henrissat, B. (2014). The carbohydrate-active enzymes database (CAZy) in 2013. Nucleic Acids Res. 42, D490-D495. doi: 10.1093/nar/gkt1178

Lonsdale, P. F., and Becker, K. (1985). Hydrothermal plumes, hot springs, and conductive heat flow in the southern trough of the Guaymas Basin. Earth Planet. Sci. Lett. 73, 211-225. doi: 10.1016/0012-821X(85)90070-6

Ludwig, W., Strunk, O., Westram, R., Richter, L., Meier, H., Yadhukumar, et al. (2004). ARB: a software environment for sequence data. Nucleic Acids Res. 32, 1363-1371. doi: 10.1093/nar/gkh293

Markowitz, V. M., Ivanova, N. N., Szeto, E., Palaniappan, K., Chu, K., Dalevi, D., et al. (2008). IMG/M: a data management and analysis system for metagenomes. Nucleic Acids Res. 36, D534-D538. doi: 10.1093/nar/gkm869

McCollom, T. M. (2000). Geochemical constraints on primary productivity in submarine hydrothermal vent plumes. Deep Sea Res. I Oceanogr. Res. Pap. 47, 85-101. doi: 10.1016/S0967-0637(99)00048-5

Parks, D. H., Imelfort, M., Skennerton, C. T., Hugenholtz, P., and Tyson, G. W. (2015). CheckM: assessing the quality of microbial genomes recovered from isolates, single cells, and metagenomes. Genome Res. 25, 1043-1055. doi: 10.1101/gr.186072.114

Petersen, T. N., Brunak, S., von Heijne, G., and Nielsen, H. (2011). SignalP 4.0: discriminating signal peptides from transmembrane regions. Nat. Methods 8, 785-786. doi: 10.1038/nmeth.1701

Quast, C., Pruesse, E., Yilmaz, P., Gerken, J., Schweer, T., Yarza, P., et al. (2013). The SILVA ribosomal RNA gene database project: improved data processing and web-based tools. Nucleic Acids Res. 41, D590-D596. doi: 10.1093/nar/gks1219

Rawlings, N. D., Waller, M., Barrett, A. J., and Bateman, A. (2014). MEROPS: the database of proteolytic enzymes, their substrates and inhibitors. Nucleic Acids Res. 42, D503-D509. doi: 10.1093/nar/gkt953

Schlesner, H., Rensmann, C., Tindall, B. J., Gade, D., Rabus, R., Pfeiffer, S., et al. (2004). Taxonomic heterogeneity within the Planctomycetales as derived by DNA-DNA hybridization, description of Rhodopirellula baltica gen. nov., sp nov., transfer of Pirellula marina to the genus Blastopirellula gen. nov as Blastopirellula marina comb. nov and emended description of the genus Pirellula. Int. J. Syst. Evol. Microbiol. 54, 1567-1580.

Sheik, C. S., Jain, S., and Dick, G. J. (2014). Metabolic flexibility of enigmatic SAR324 revealed through metagenomics and metatranscriptomics. Environ. Microbiol. 16, 304-317. doi: 10.1111/1462-2920.12165

Shi, Y. M., Tyson, G. W., and DeLong, E. F. (2009). Metatranscriptomics reveals unique microbial small RNAs in the ocean's water column. Nature 459, 266-269. doi: $10.1038 /$ nature 08055

Steen, A. D., Ziervogel, K., Ghobrial, S., and Arnosti, C. (2012). Functional variation among polysaccharide-hydrolyzing microbial communities in the Gulf of Mexico. Mar. Chem. 138, 13-20. doi: 10.1016/j.marchem.2012.06.001

Sunamura, M., Higashi, Y., Miyako, C., Ishibashi, J., and Maruyama, A. (2004). Two bacteria phylotypes are predominant in the Suiyo seamount hydrothermal plume. Appl. Environ. Microbiol. 70, 1190-1198. doi: 10.1128/AEM.70.2.11901198.2004

Sylvan, J. B., Pyenson, B. C., Rouxel, O., German, C. R., and Edwards, K. J. (2012). Time-series analysis of two hydrothermal plumes at 9 degrees 50'N East Pacific Rise reveals distinct, heterogeneous bacterial populations. Geobiology 10, 178-192. doi: 10.1111/j.1472-4669.2011.00315.x

Tamura, K., Stecher, G., Peterson, D., Filipski, A., and Kumar, S. (2013). MEGA6: molecular evolutionary genetics analysis version 6.0. Mol. Biol. Evol. 30, 27252729. doi: 10.1093/molbev/mst197

Vondamm, K. L., Edmond, J. M., Measures, C. I., and Grant, B. (1985). Chemistry of submarine hydrothermal solutions at Guaymas Basin, Gulf of California. Geochim. Cosmochim. Acta 49, 2221-2237. doi: 10.1016/0016-7037(85)90223-6

Wagner, M., and Horn, M. (2006). The Planctomycetes, Verrucomicrobia, Chlamydiae and sister phyla comprise a superphylum with biotechnological and medical relevance. Curr. Opin. Biotechnol. 17, 241-249. doi: 10.1016/j.copbio.2006.05.005

Winn, C. D., Karl, D. M., and Massoth, G. J. (1986). Microorganisms in Deep-Sea Hydrothermal Plumes. Nature 320, 744-746. doi: 10.1038/320744a0 
Woebken, D., Teeling, H., Wecker, P., Dumitriu, A., Kostadinov, I., Delong, E. F., et al. (2007). Fosmids of novel marine Planctomycetes from the Namibian and Oregon coast upwelling systems and their cross-comparison with planctomycete genomes. ISME J. 1, 419-435. doi: 10.1038/ismej.2007.63

Youssef, N. H., and Elshahed, M. S. (2014). "The phylum planctomycetes," in The Prokaryotes Other Major Lineages of Bacteria and The Archaea, ed. E. Rosenberg (Heidelberg: Springer), 760-810.

Yu, N. Y., Wagner, J. R., Laird, M. R., Melli, G., Rey, S., Lo, R., et al. (2010). PSORTb 3.0: improved protein subcellular localization prediction with refined localization subcategories and predictive capabilities for all prokaryotes. Bioinformatics 26, 1608-1615. doi: 10.1093/bioinformatics/btq249

Zhang, H., Sekiguchi, Y., Hanada, S., Hugenholtz, P., Kim, H., Kamagata, Y., et al. (2003). Gemmatimonas aurantiaca gen. nov., sp nov., a gram-negative, aerobic, polyphosphate-accumulating micro-organism, the first cultured representative of the new bacterial phylum Gemmatimonadetes phyl. nov. Int. J. Syst. Evol. Microbiol. 53, 1155-1163. doi: 10.1099/ijs.0.02520-0

Conflict of Interest Statement: The authors declare that the research was conducted in the absence of any commercial or financial relationships that could be construed as a potential conflict of interest.

Copyright (c) $2016 \mathrm{Li}$, Jain and Dick. This is an open-access article distributed under the terms of the Creative Commons Attribution License (CC BY). The use, distribution or reproduction in other forums is permitted, provided the original author(s) or licensor are credited and that the original publication in this journal is cited, in accordance with accepted academic practice. No use, distribution or reproduction is permitted which does not comply with these terms. 